\title{
Ultra-widefield fluorescein angiography of white without pressure
}

This article was published in the following Dove Press journal:

Clinical Ophthalmology

23 April 2013

Number of times this article has been viewed

\section{Anton Orlin \\ Aalya Fatoo \\ Joshua Ehrlich \\ Donald J D'Amico \\ RV Paul Chan \\ Szilárd Kiss}

Department of Ophthalmology, Weill Cornell Medical College, New York, NY, USA
Correspondence: Anton Orlin Weill Cornell Medical College, I 305 York Avenue, I I th Floor, Room Y I I.34, New York, NY, I002I, USA

Tel + I 6469622540

Fax + I 6469620602

Email ano9028@med.cornell.edu
Purpose: To describe ultra-widefield fluorescein angiography (UWFA) findings in eyes with white without pressure (WWOP) and in eyes without any obvious peripheral chorioretinal disease, and to determine if a difference exists between these two groups.

Methods: A retrospective review of 379 eyes undergoing diagnostic UWFA using the Optos 200Tx imaging system. Eyes were excluded if the quality of the color photograph or UWFA prevented reliable evaluation. Eyes were also excluded if there was any evidence of peripheral retinal or choroidal disease, which was thought to have an effect on UWFA (eg, peripheral background diabetic or hypertensive retinopathy, vein occlusion, or any other peripheral vascular disorder). Eyes were determined to have WWOP, based on a dilated fundus examination and color fundus photography that contained areas of peripheral retinal whitening consistent with the diagnosis. UWFA was evaluated by trained masked graders, and determined to have or not have peripheral vascular leakage and/or staining.

Results: Of the 379 eyes evaluated, 45 eyes were included in the study. Twelve eyes were determined to have peripheral WWOP; 33 eyes did not have WWOP on examination or color fundus photography. Three common UWFA peripheral patterns were visualized. Eyes with and without WWOP were grouped into one of three patterns. The majority of eyes without WWOP demonstrated UWFA pattern one $(69.7 \%)$, while those in the WWOP group demonstrated pattern three $(50 \%)$. The distribution of UWFA patterns is statistically different between those with and without WWOP $(P=0.002)$. In eyes without WWOP, in patients with no documented systemic microvascular disease (diabetes, hypertension), $71.4 \%$ of eyes had UWFA pattern one while $14.3 \%$ had both patterns two and three.

Conclusion: This study is one of the first to specifically evaluate peripheral vascular leakage/ staining in eyes with WWOP as well as in eyes without any obvious peripheral chorioretinal disease. We demonstrate that a significant portion of WWOP eyes exhibit peripheral findings on UWFA (pattern one) compared to eyes without WWOP. Importantly, even in eyes that are apparently unremarkable in the periphery on exam and color photography, UWFA can still show peripheral vascular abnormalities. These results warrant further investigation.

Keywords: ocular imaging, ptos imaging, retina periphery

\section{Introduction}

Retinal white with pressure (WWP) was first described by Schepens ${ }^{1}$ as an area of retinal whitening which blurs the underlying choroid under scleral depression; white without pressure (WWOP) is a similar phenomenon that occurs without the scleral indentation. The exact pathogenesis remains unknown, and although controversial, it has been thought of as an optical phenomenon associated with vitreous traction or adhesion to the overlying retinal surface. ${ }^{2,3}$ If the light, during indirect ophthalmoscopy, 
is tangential to the more dense vitreous fibers, a white appearance and reflex results to the underlying retina.,4

WWOP has been reported to occur in up to $30 \%$ of the general population, is more common in myopic patients, ${ }^{5}$ and with increasing age. ${ }^{6}$ As in conditions with increased vitroretinal adhesion and traction, WWOP may potentially predispose to the development of retinal breaks, ${ }^{7}$ although this has not been consistently demonstrated. ${ }^{5}$

WWOP occurs in the far periphery, and is therefore technically difficult to capture with standard photography or fluorescein angiography (FA). Traditional cameras employ retinal photography that is able to view approximately 30 to 50 degrees of the retina at one time. Using seven-standard fields (7SF) protocol, in which seven photographed areas of the retina are combined, gives us a broader view and 75 to 100 degrees of visualization. ${ }^{8,9}$ This protocol, however, can be technically more challenging for the patient and photographer, and the far peripheral retina is still not well visualized. With the advent of ultra-widefield fluorescein angiography (UWFA), as with the Optos 200Tx imaging system (Optos PLC, Dunfermline, Scotland), it is now possible to view up to 200 degrees of retina in a single photograph. ${ }^{8,10}$ This rapid acquisition system requires less photographic expertise and less patient cooperation than 7SF. It also allows for ultra-widefield color imaging and UWFA of nearly the entire retina during which the peripheral retina is captured simultaneously without the need for patient refixation.

Since its development nearly 50 years ago, FA remains instrumental in the evaluation of retinal diseases and in further characterizing pathologic changes especially in the retinal vasculature. ${ }^{8}$ The development of ultra-widefield imaging has enabled us to obtain peripheral fluorescein angiograms in eyes with WWOP.

The purpose of our study was to describe UWFA in eyes with WWOP, which may help further define this pathogenesis. This is the first such study specifically to evaluate patients with WWOP. We also aimed to describe peripheral UWFA in otherwise normal eyes without any obvious peripheral chorioretinal pathology.

\section{Methods}

This retrospective review was approved by the Institutional Review Board of Weill Cornell Medical College (WCMC, [New York, NY, USA]). The research adhered to the tenets of the Declaration of Helsinki and was conducted in accordance with the Health Insurance Portability and Accountability Act regulations. All patients who underwent UWFA using the Optos 200Tx imaging system at WCMC between 2008 and 2011 were retrospectively reviewed and included in this study.

Eyes were excluded if the quality of the color photograph or UWFA prevented reliable evaluation. Eyes were also excluded if they contained any peripheral pathology on dilated fundus exam and color photography (such as peripheral drusen, background diabetic or hypertensive retinopathy, vein occlusion, or other vascular disease that may have a potential effect on the appearance of the peripheral UWFA). Baseline information was recorded from the chart review, including the patient's age, gender, and past medical history. Eyes were determined to have WWOP based on dilated fundus examination and color fundus photography and contained areas of peripheral retinal whitening consistent with the diagnosis. We sought to characterize UWFA findings in our WWOP group and in those without WWOP, and to compare UWFA between the two groups.

\section{Statistical analysis}

The Mann-Whitney $U$ test was used to test for a difference in age between groups. The Fisher Exact test was used to test for differences in the distribution of categorical variables among patients with and without WWOP. All statistical tests were two-sided with a significance threshold of $P \leq 0.05$. Statistical analyses were performed using Stata 11/IC (Stata Corp, College Station, TX, USA). The Fisher Exact test was used to determine whether any statistically meaningful difference existed in the distribution of indications for which WWOP and control eyes underwent FA.

\section{Results}

A total of 379 consecutive UWFAs were reviewed; 45 eyes were included in the study; 12 eyes were determined to have peripheral WWOP; and 33 eyes had no WWOP or apparent peripheral chorioretinal pathology. Refer to Table 1 for baseline characteristics. Table 2 details the reasoning for obtaining UWFA among the eyes in this cohort. There was no statistically significant difference in the distribution of reasons for obtaining FA between the WWOP and control eyes $(P=0.41)$. Patients on average were older in the group without WWOP (54.9 years versus 46.5 years, $P=0.02$ ) and the cohort ranged in age from 26 to 79 years of age. Seventy-five percent of the patients in the WWOP group were females compared to $41.2 \%$ in the group without WWOP $(P=0.1) ; 66.7 \%$ of patients with WWOP had a documented history of either diabetes or hypertension compared to $78.8 \%$ in the group without 
Table I Baseline characteristics

\begin{tabular}{llll}
\hline & $\begin{array}{l}\text { White without } \\
\text { pressure eyes }\end{array}$ & Control eyes & P-value \\
\hline $\begin{array}{l}\text { Number of eyes } \\
\text { Age of patient (years) }\end{array}$ & 12 & 33 & \\
$\%$ female (number) & $75 \%(9)$ & 54.9 & 0.02 \\
$\%$ right eye (n) & $50 \%(6)$ & $41.2 \%$ & 0.1 \\
PMH: diabetes or & $66.7 \%(8)$ & $51.5 \%(17)$ & 1.0 \\
hypertension & & $78.8 \%(26)$ & 0.4 \\
\hline
\end{tabular}

Abbreviation: $\mathrm{PMH}$, past medical history.

WWOP $(P=0.4)$. All eyes with diabetic or hypertensive retinopathy extending peripheral to the posterior pole were excluded. The right eye was evaluated in $50 \%$ of eyes in the WWOP group and in $51.5 \%$ in the group without WWOP $(P=1.0)$.

Refer to Figure 1A and B (color fundus photographs with WWOP) and Figure 2 (color fundus photograph without WWOP or peripheral pathology). Based on all UWFAs reviewed, three common peripheral patterns were visualized. Eyes with and without WWOP were grouped into one of three patterns. Pattern one (Figure 3A and B) demonstrated a decreasing amount of hyperfluorescence as the image progressed to the periphery from the posterior pole. Pattern two (Figure 4A-C) demonstrated an area of increased hyperfluorescence peripherally which decreased as the image progressed posteriorly, blending into the rest of the image. Pattern three (Figure 5A-C) is similar to pattern two, but also contained multiple pinpoint areas of

Table 2 Reason for obtaining UWFA

\begin{tabular}{|c|c|c|}
\hline & $\begin{array}{l}\text { WWOP group } \\
(n=12) \\
n(\%)\end{array}$ & $\begin{array}{l}\text { Control eyes } \\
(n=33) \\
n(\%)\end{array}$ \\
\hline Uveitis & $5(4 I .7 \%)$ & $5(15.2 \%)$ \\
\hline Uncontrolled hypertension & $2(16.7 \%)$ & $6(18.2 \%)$ \\
\hline Elevated $\mathrm{HbA}{ }_{1 \mathrm{c}}$, no BDR & $3(25 \%)$ & $10(30.3 \%)$ \\
\hline Sickle cell retinopathy & I (8.3\%) & $0(0 \%)$ \\
\hline $\begin{array}{l}\text { Central serous retinopathy } \\
\text { (posterior macular RPE changes) }\end{array}$ & I (8.3\%) & $2(6.1 \%)$ \\
\hline $\begin{array}{l}\text { Vein occlusion in contralateral } \\
\text { eye (involved eye excluded) }\end{array}$ & $0(0 \%)$ & $4(12.1 \%)$ \\
\hline $\begin{array}{l}\text { Myopia, rule out choroidal } \\
\text { neovascular membrane or } \\
\text { reasons for subjective } \\
\text { "vision loss" }\end{array}$ & $0(0 \%)$ & $2(6.1 \%)$ \\
\hline Retinal hemorrhages & $0(0 \%)$ & $3(9.1 \%)$ \\
\hline $\begin{array}{l}\text { Visual field loss, rule out ocular } \\
\text { ischemia }\end{array}$ & $0(0 \%)$ & I (3.0\%) \\
\hline
\end{tabular}

Note: $P$-value $=0.4 \mathrm{I}$ (distribution in the reasoning for obtaining UWFA between the WWOP and control groups).

Abbreviations: $B D R$, background diabetic retinopathy; $\mathrm{Hb}_{\mathrm{lc}}$, hemoglobin $\mathrm{A}_{\mathrm{lc}}$; RPE, retinal pigment epithelium; UWFA, ultra-widefield fluorescein angiography; WWOP, white without pressure.
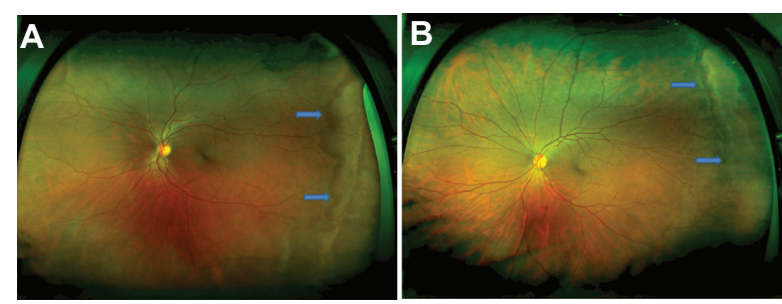

Figure I (A and B) Color fundus photograph of white without pressure.

hyperfluorescence in the far periphery. Both patterns two and three appeared to correspond to the areas of WWOP on exam and color photo (Figures $4 \mathrm{C}$ and $5 \mathrm{C}$ ). No other chorioretinal findings such as drusen, hemorrhages, atrophy, or scarring were visualized in this region. Table 3 summarizes the UWFA pattern distribution among the eyes with and without WWOP. The majority of eyes in the WWOP group demonstrate UWFA pattern type three $(50 \%, \mathrm{n}=6)$ correlating with the areas of WWOP, while only $6.1 \%(n=2)$ of eyes without WWOP demonstrate this pattern. Twenty-five percent $(n=3)$ of WWOP eyes demonstrate UWFA pattern type one compared to $69.7 \%$ $(\mathrm{n}=23)$ of eyes without WWOP; 25\% $(\mathrm{n}=3)$ of WWOP eyes demonstrate pattern type two compared to $24.2 \%$ $(n=8)$ of the eyes without WWOP. The distribution of UWFA patterns is statistically different between those with and without WWOP $(P=0.002)$.

In the eyes without WWOP or apparent peripheral chorioretinal disease, seven eyes belonged to patients with no known systemic vascular disease such as hypertension or diabetes (see Table 4); 71.4\% $(n=5)$ of these eyes displayed UWFA pattern type one, while $14.3 \%(\mathrm{n}=1)$ demonstrated UWFA pattern types two and three.

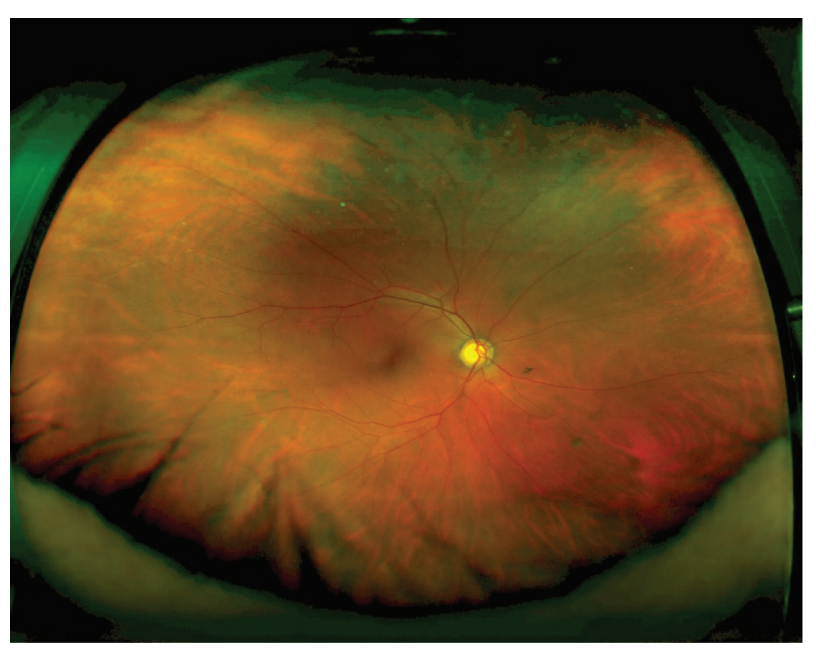

Figure 2 Color fundus photograph of an eye without white without pressure or peripheral pathology. 

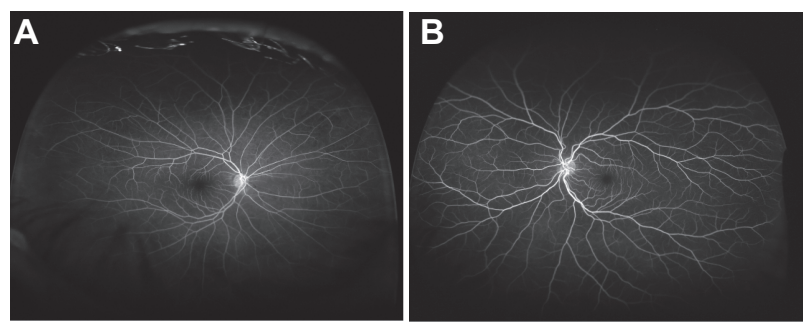

Figure 3 (A and $\mathbf{B}$ ) Ultra-widefield fluorescein angiography pattern one.

\section{Discussion}

UWFA was first described in 2004 by Friberg and Forrester ${ }^{11}$ and Friberg et $\mathrm{al}^{12}$ and has been a reliable method of retinal evaluation. It allows for visualization of the peripheral retina and we utilized this tool to evaluate eyes with and without WWOP. To the best of our knowledge, this is one of the first studies to specifically evaluate peripheral vascular fluorescein patterns/leakage in eyes with WWOP as well as in eyes with no obvious peripheral chorioretinal disease.

Based on all UWFAs reviewed, three common peripheral patterns were described. Pattern one revealed a decreasing amount of hyperfluorescence as the image progressed to the periphery from the posterior pole. Pattern two demonstrated an area of increased hyperfluorescence peripherally which decreased posteriorly, blending into the rest of the image. Pattern three is similar to pattern two, but also contained multiple pinpoint areas of hyperfluorescence in the far periphery. The distribution of UWFA patterns is statistically different between those with and without WWOP $(P=0.002)$. A larger percentage of eyes without
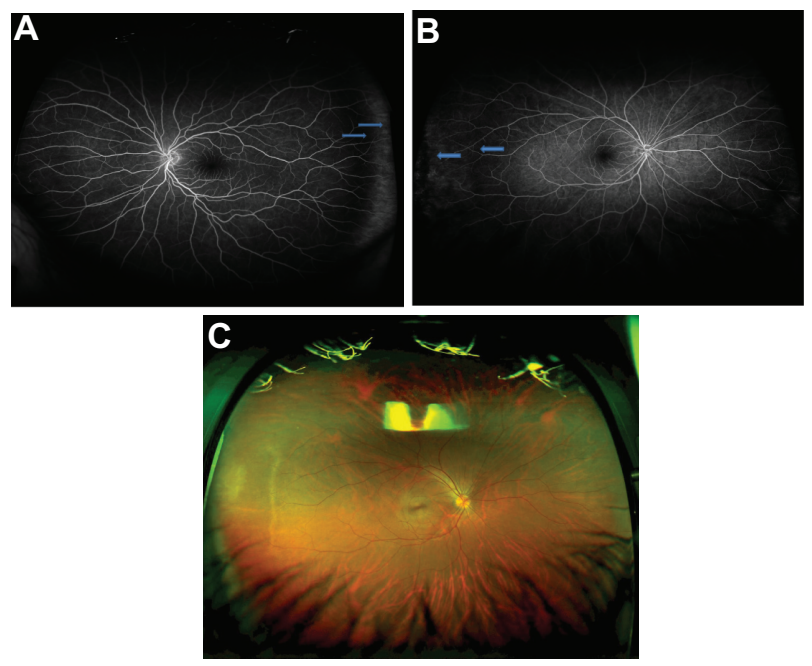

Figure 4 (A and B) Ultra-widefield fluorescein angiography pattern two. (C) Color photo corresponding to Figure 4B (ultra-widefield fluorescein angiography pattern two).
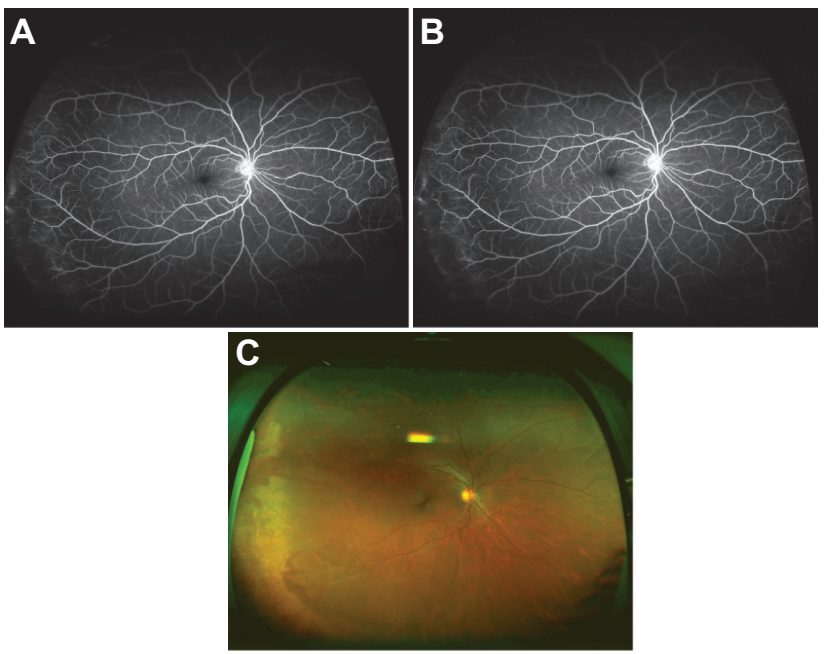

Figure 5 (A and $\mathbf{B}$ ) Ultra-widefield fluorescein angiography pattern three. (C) Color photo corresponding to Figure $5 \mathrm{~A}$ and $\mathrm{B}$.

WWOP displayed pattern one when compared to the eyes with WWOP. Conversely, more eyes with WWOP displayed pattern three, a localized peripheral hyperfluorescence with additional pinpoint areas of hyperfluorescence. It is unclear exactly what these UWFA patterns represent, but the increased peripheral hyperfluorescence in patterns two and three possibly represent areas of peripheral leakage/staining of fluorescein dye.

Although the exact pathogenesis of WWOP remains unknown, it is thought to be an optical phenomenon associated with vitreous traction to the overlying retinal surface. ${ }^{2,3}$ Additionally, the nerve fiber layer overlying the capillaries thins in the periphery, resulting in a decreased cushion for the blood vessels. Traction or tension will result in a greater force exerted on the vessels in these areas, possibly resulting in diminished blood flow, and the blanching of the area. ${ }^{13,14}$ The significance of the small pinpoint areas on UWFA (pattern three) findings is unclear, but could represent areas of peripheral leakage from overlying traction. This could be consistent with and further support the above theory on the pathogenesis of WWOP, and its association with increased vitreous traction. As in eyes with vitreous traction of any etiology, it may be necessary to follow those with WWOP more closely to monitor for development of

Table 3 Fluorescein angiography characteristics

\begin{tabular}{llll}
\hline & $\begin{array}{l}\text { White without } \\
\text { pressure eyes }\end{array}$ & Control eyes & P-value \\
\hline$\%$ pattern I (n) & $25 \%(3)$ & $69.7 \%(23)$ & 0.002 \\
$\%$ pattern 2 (n) & $25 \%(3)$ & $24.2 \%(8)$ & \\
$\%$ pattern 3 (n) & $50 \%(6)$ & $6.1 \%(2)$ & \\
\hline
\end{tabular}


Table 4 Fluorescein angiography characteristics in control eyes only, based on PMH

\begin{tabular}{lll}
\hline & $\begin{array}{l}\text { PMH of diabetes or } \\
\text { hypertension }\end{array}$ & $\begin{array}{l}\text { No PMH of diabetes or } \\
\text { hypertension }\end{array}$ \\
\hline$\%$ pattern I (n) & $71.4 \%(5)$ & $69.2 \%(18)$ \\
$\%$ pattern 2 (n) & $14.3 \%(1)$ & $26.9 \%(7)$ \\
$\%$ pattern 3 (n) & $14.3 \%(I)$ & $3.9 \%(1)$ \\
\hline
\end{tabular}

Abbreviation: $\mathrm{PMH}$, past medical history.

retinal tear and detachment, although this association has not been clearly demonstrated in the past. . $^{5,7}$

The vast majority of eyes without WWOP or peripheral chorioretinal pathology demonstrated UWFA pattern one. This held true in subgroup analysis whether eyes were from patients with or without a documented history of systemic hypertension or diabetes (Table 4). Pattern one likely represents the UWFA more highly associated with normal vascularized control eyes which do not have peripheral pathology such as WWOP. It is important to note, that these eyes without WWOP or other apparent peripheral pathology, still demonstrated UWFA patterns two and three regardless of whether there was a past history of diabetes or hypertension (Table 4). Therefore, when interpreting UWFA, one must not jump to the conclusion that an eye has peripheral chorioretinal or vascular disease when suspected peripheral leakage/staining on UWFA (patterns two or three) is seen. This interpretation is applicable to various diseases in which UWFA plays an important role, such as with diabetes, hypertension, vasculitis, chorioretinitis, or familial exudative vitreoretinopathy.

Several important limitations to our study include those biases intrinsic to a retrospective one. We did attempt to identify any baseline differences between eyes with and without WWOP. On average, eyes without WWOP were older than with WWOP $(P=0.02)$. It is unclear if this would affect the UWFA findings, as UWFA correlated to age has never been studied previously. The difference in age, however, was not dramatic with a mean of 46.5 years in the WWOP group compared to 54.9 years in the group without WWOP. We attempted to minimize any effect diabetes or hypertension would have on the UWFA findings. All eyes diagnosed with peripheral diabetic or hypertensive retinopathy were excluded. There was no baseline difference regarding past documented history of diabetes or hypertension in eyes with and without WWOP. Since the ability to easily take UWFA photographs is relatively new, another limitation is the size of our cohort, although a statistically significant difference was observed among UWFA patterns between the two groups. Future investigation with a larger cohort should be made to further define these differences and whether baseline characteristics (Tables 1 and 2) do play a role in the peripheral fluorescein patterns on UWFA.

Besides for WWOP, eyes with peripheral chorioretinal findings on fundus examination (eg, diabetic retinopathy, drusen, chorioretinal scarring, and vein occlusion) were excluded. Eyes in our cohort obtained UWFA for various reasons (Table 2). Although eyes with apparent peripheral diabetic or hypertensive retinopathy were excluded, it is unclear what effect uncontrolled hypertension or an uncontrolled hemoglobin $\mathrm{A}_{1 \mathrm{c}}$ would have on the peripheral retinal circulation and UWFA. It is also unclear what effect diseases traditionally thought to involve the posterior pole, such as central serous retinopathy, would have on the peripheral UWFA. This uncertainty warrants further investigation, although there was no statistically significant difference in the distribution of reasons for obtaining FA between the WWOP and control eyes $(P=0.41)$. Therefore we believe that these baseline characteristics would have no significant influence on our results, and the differences in UWFA between the two groups. Additionally, in the WWOP eyes, UWFA patterns two and three appeared to correspond to areas of WWOP on color photos (Figures 4C and 5C). No other chorioretinal findings such as drusen, hemorrhages, atrophy, or scarring were visualized in this region.

To conclude, to our knowledge, this study is the first to describe UWFA in eyes with WWOP, and further characterize peripheral UWFA findings in eyes without WWOP or other apparent peripheral chorioretinal pathology. Our findings suggest that there are differences in UWFA among eyes with and without WWOP, which may help further define the pathogenesis of WWOP. In addition, although eyes with an apparently normal peripheral examination largely demonstrate UWFA pattern one, UWFA can still show peripheral vascular abnormalities (patterns two and three). Further investigation is necessary to determine the clinical significance of the different UWFA patterns. Future studies with a larger cohort should also determine whether age, uncontrolled hypertension, and elevated $\mathrm{HbA}_{1 \mathrm{c}}$ levels affect UWFA, and whether diseases traditionally thought to affect the posterior pole such as central serous retinopathy affect the peripheral vasculature.

\section{Disclosure}

Szilard Kiss is a consultant for optos. The other authors report no conflicts of interest in this work. 


\section{References}

1. Schepens CL. Retinal detachment; diagnostic and prognostic factors as found preoperative examination. Trans Am Acad Ophthalmol Otolaryngol. 1952;56:398-412.

2. Watzke R. The ophthalmic sign, "white with pressure": A clinicopathologic correlation. Arch Ophthalmol. 1961;66:812-823.

3. Steidel SM, Hartnett ME. Clinical pathways in vitreoretinal disease. New York: Thieme Medical Publishers, Inc; 2003.

4. Green W, Sebag J. Vitreoretinal interface. In: Ryan S, editor. Retina, 3rd ed. St Louis: Mosby; 2001:1882-1960.

5. Shukla M, Anuja OP. White with pressure (WWP) and white without pressure (WWOP) lesions. Indian J Ophthalmol. 1982;30:129-132.

6. Rutnin U, Schepens CL. Fundus appearance in normal eyes. 3. Peripheral degenerations. Am J Ophthalmol. 1967;64:1040-1062.

7. Freeman HM. Fellow eyes of giant retinal breaks. Trans Am Ophthalmol Soc. 1978;76:343-382.

8. Wessel MM, Aaker GD, Parlitsis G, Cho M, D'amico DJ, Kiss S. Ultra-wide-field angiography improves the detection and classification of diabetic retinopathy. Retina. 2012;32:785-791.
9. Diabetic Retinopathy Study Research Group: Design methods and baseline results. DRS report no 6. Invest Ophthalmol. 1991;21:149-209.

10. Wessel MM, Nair N, Aaker GD, Ehrlich JR, D'Amico DJ, Kiss S. Peripheral retinal ischaemia, as evaluated by ultra-widefield fluorescein angiography, is associated with diabetic macular oedema. $\mathrm{Br} J$ Ophthalmol. 2012;96:694-698.

11. Friberg TR, Forrester JV. Ultrawide angle (200+) fluorescein angiography using a modified Optos Panoramic200TM Imaging System. Invest Ophthalmol Vis Sci. 2004;45:ARVO E-Abstract 3001.

12. Friberg TR, Gupta A, Yu J, et al. Ultrawide angle fluorescein angiographic imaging: a comparison to conventional digital acquisition systems. Ophthalmic Surg Lasers Imaging. 2008;39:304-311.

13. Kolb $\mathrm{H}$. The neural organization of the human retina. In: Heckenlively JR, Arden GB, editors. Principles and Practices of Clinical Electrophysiology of Vision. St Louis: Mosby Year Book Inc; 1991:25-52.

14. Henkind P, Hansen RI, Szalay J. Ocular circulation. In: Records RE, editor. Physiology of the Human Eye and Visual System. New York: Harper and Row. 1979:98-155.

\section{Clinical Ophthalmology}

\section{Publish your work in this journal}

Clinical Ophthalmology is an international, peer-reviewed journal covering all subspecialties within ophthalmology. Key topics include: Optometry; Visual science; Pharmacology and drug therapy in eye diseases; Basic Sciences; Primary and Secondary eye care; Patient Safety and Quality of Care Improvements. This journal is indexed on

Submit your manuscript here: http://www.dovepress.com/clinical-ophthalmology-journal

\section{Dovepress}

PubMed Central and CAS, and is the official journal of The Society of Clinical Ophthalmology (SCO). The manuscript management system is completely online and includes a very quick and fair peer-review system, which is all easy to use. Visit http://www.dovepress.com/ testimonials.php to read real quotes from published authors. 